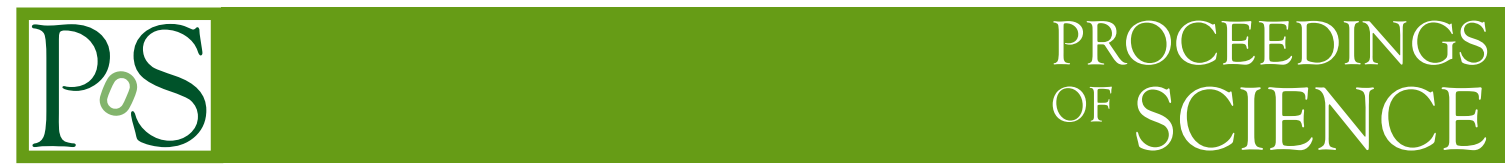

\title{
(Semi-) leptonic charmed meson decays at BESIII
}

\author{
Huijing $\mathbf{L i}^{a, b, 1, *}$ \\ ${ }^{a}$ Henan Normal University, \\ No. 46, East of Construction Road, Xinxiang, Henan, China \\ ${ }^{b}$ Demonstration Center for Experimental Physics Education (Henan Normal University), \\ No. 46, East of Construction Road, Xinxiang, Henan, China \\ E-mail: lihuijing@htu.edu.cn
}

BESIII has collected $2.93 \mathrm{fb}^{-1}$ and $6.32 \mathrm{fb}^{-1}$ of $e^{+} e^{-}$collision data samples at the center-of-mass energies 3.773 and $4.178-4.226 \mathrm{GeV}$, respectively. We report recent measurements of the (semi-) leptonic decays $D_{s} \rightarrow \ell^{+} v_{\ell}(\ell=\mu, \tau)$ and $D_{(s)} \rightarrow X \ell^{+} v_{\ell}\left[X=\eta, K_{1}(1270)\right.$ or denotes generic decays, and $\ell=e, \mu]$. The decay constant $f_{D_{s}^{+}}$, the semileptonic form factor $f_{+}^{\eta}(0)$ and the CKM matrix elements $\left|V_{c d(s)}\right|$ are determined precisely. These results are important to verify the theoretical calculations of $f_{D_{s}^{+}}$and $f_{+}^{\eta}(0)$, and the unitarity of the CKM matrix. Precision tests of lepton-flavor universality with (semi-) leptonic $D_{(s)}$ decays are also performed.

10th International Workshop on Charm Physics (CHARM2020),

31 May - 4 June, 2021

Mexico City, Mexico - Online

\footnotetext{
${ }^{1}$ For the BESIII Collaboration.

* Speaker
} 


\section{Introduction}

In the standard model (SM), due to lepton flavor universality (LFU), the ratio of decay widths [1] for the purely leptonic decays of a charmed meson with different generation leptons should only depend on the masses of the leptons and the charmed meson, and it is expected to have the same branching fraction (BF) for the charmed meson decaying to the same hadronic final states and different generation leptons. However, the hints of LFU violation have been observed in the semileptonic $B$ decays recently [2]. Thus, it will be of great interest to search for LFU in the purely leptonic and semi-leptonic $D$ decays with high precision. Besides, measurements of these decays will also provide the accurate basis for deducing the decay constant $f_{D_{s}^{+}}$or form factor $f_{+}(0)$, and Cabibbo-Kobayashi-Maskawa (CKM) matrix elements $\left|V_{c d(s)}\right|$, which are helpful to calibrate the Lattice quantum chromodynamics (LQCD) calculations and test the unitarity of the CKM matrix, respectively.

Benefiting from the $D \bar{D}$ and $D_{s}^{* \pm} D_{s}^{\mp}$ pairs produced in $e^{+} e^{-}$collision at center-of-mass energies of $\sqrt{s}=3.773 \mathrm{GeV}$ and $\sqrt{s}=4.178-4.226 \mathrm{GeV}$ [3], respectively, a double-tag (DT) technique pioneered by the Mark III experiments [4] can be employed to measure the absolute BF of $D_{(s)}$ decays in BESIII experiment [5]. A DT signal is the purely leptonic or semi-leptonic signal $D_{(s)}$ candidate accompanied by a fully reconstruction of the other $D_{(s)}$ meson. Throughout the text, charge-conjugate modes are implicitly assumed, unless otherwise stated.

\section{Purely leptonic $D_{s}^{+}$decays}

\section{1 $D_{s}^{+} \rightarrow \tau^{+} v_{\tau}$ via $\tau^{+} \rightarrow e^{+} v_{e} \bar{v}_{\tau}$}

Since the massless neutrinos are undetected, the variable of $E_{\text {extra }}^{\text {tot }}$ has been adopted to analyze the signal $D_{s}^{+} \rightarrow \tau^{+} v_{\tau}$ via $\tau^{+} \rightarrow e^{+} v_{e} \bar{v}_{\tau}$ [6], where $E_{\text {extra }}^{\text {tot }}$ denotes the total energy of the good electromagnetic calorimeter (EMC) showers excluding those associated with the fully reconstructed $D_{s}^{-}$candidate and those within $5^{\circ}$ of the initial direction of the positron. The signal yields are determined in the region $E_{\text {extra }}^{\text {tot }}<0.4 \mathrm{GeV}$ by statistically subtracting the backgrounds extrapolated from the fits to $E_{\text {extra }}^{\text {tot }}$ in $E_{\text {extra }}^{\text {tot }}>0.6 \mathrm{GeV}$. The BF of $D_{s}^{+} \rightarrow \tau^{+} v_{\tau}$ is determined to be $\mathcal{B}_{D_{s}^{+} \rightarrow \tau^{+} v_{\tau}}=$ $\left(5.27 \pm 0.10_{\text {stat. }} \pm 0.12_{\text {syst. }}\right) \%$, which is the most precise result to date.

\section{2 $D_{s}^{+} \rightarrow \tau^{+} v_{\tau}$ via $\tau^{+} \rightarrow \pi^{+} \pi^{0} \bar{v}_{\tau}$}

In order to obtain the signal yield in the analysis of $D_{s}^{+} \rightarrow \tau^{+} v_{\tau}$ via $\tau^{+} \rightarrow \pi^{+} \pi^{0} \bar{\nu}_{\tau}$ [7], a simultaneous fit to the missing mass square $\left(\mathrm{MM}^{2}\right)$ is performed for the data samples at $\sqrt{s}=$ 4.178-4.226 GeV, sharing with a common $\mathcal{B}_{D_{s}^{+} \rightarrow \tau^{+} \nu_{\tau}}$. Here, the missing means the information of the undetected neutrinos. The signal yield is estimated to be $(1745 \pm 84)$, and the measured $\mathcal{B}_{D_{s}^{+} \rightarrow \tau^{+} v_{\tau}}=\left(5.29 \pm 0.25_{\text {stat. }} \pm 0.20_{\text {syst. }}\right) \%$.

\section{3 $D_{s}^{+} \rightarrow \tau^{+} v_{\tau}$ via $\tau^{+} \rightarrow \pi^{+} \bar{v}_{\tau}$ and $D_{s}^{+} \rightarrow \mu^{+} v_{\mu}$}

Due to the similar masses between pion and muon, the analyses of $D_{s}^{+} \rightarrow \tau^{+} v_{\tau}$ via $\tau^{+} \rightarrow \pi^{+} \bar{v}_{\tau}$ and $D_{s}^{+} \rightarrow \mu^{+} v_{\mu}$ are studied together [3]. The sample in the signal $D_{s}^{+}$side is split into two parts based on the energy-deposit $\left(E_{\mathrm{EMC}}\right)$ properties of muons and pions in the EMC. Candidates 
with $E_{\mathrm{EMC}} \leq 300 \mathrm{MeV}$ are classified as $\mu$-like sample and the remainder as $\pi$-like sample. An unbinned simultaneous maximum likelihood fit to the two dimensional (2D) distributions are performed for the two kinds of samples, where the 2D distributions are the invariant mass of the fully reconstructed $D_{s}^{-}$and the $\mathrm{MM}^{2}$. The signal yields are obtained to be $\left(946_{-45}^{+46}\right)$ for $D_{s}^{+} \rightarrow \tau^{+} v_{\tau}$, and (2198 \pm 55$)$ for $D_{s}^{+} \rightarrow \mu^{+} v_{\mu}$, where the uncertainty are statistical only. The measured BFs are $\mathcal{B}_{D_{s}^{+} \rightarrow \tau^{+} v_{\tau}}=\left(5.21 \pm 0.25_{\text {stat. }} \pm 0.17_{\text {syst. }}\right) \times 10^{-2}$, and $\mathcal{B}_{D_{s}^{+} \rightarrow \mu^{+} v_{\mu}}=\left(5.35 \pm 0.13_{\text {stat. }} \pm 0.16_{\text {syst. }}\right) \times 10^{-3}$.

Combined the above results from BESIII measurements and the world average values [8], the ratio of the $\mathcal{B}_{D_{s}^{+} \rightarrow \tau^{+} v_{\tau}}$ over $\mathcal{B}_{D_{s}^{+} \rightarrow \mu^{+} v_{\mu}}$ is estimated to be $9.67 \pm 0.34$, which is consistent with the SM prediction of $(9.75 \pm 0.01)$.

Taking the CKM matrix element $\left|V_{c s}\right|=0.97320 \pm 0.00011$ from the global SM fit [8], or the averaged decay constant $f_{D_{s}^{+}}=249.9 \pm 0.5 \mathrm{MeV}$ of LQCD calculations [9] as input, the $f_{D_{s}^{+}}$and $\left|V_{c s}\right|$ can be determined, respectively. Figure 1 shows the comparisons of $f_{D_{s}^{+}}$and $\left|V_{c s}\right|$ between the BESIII measurements and other results.

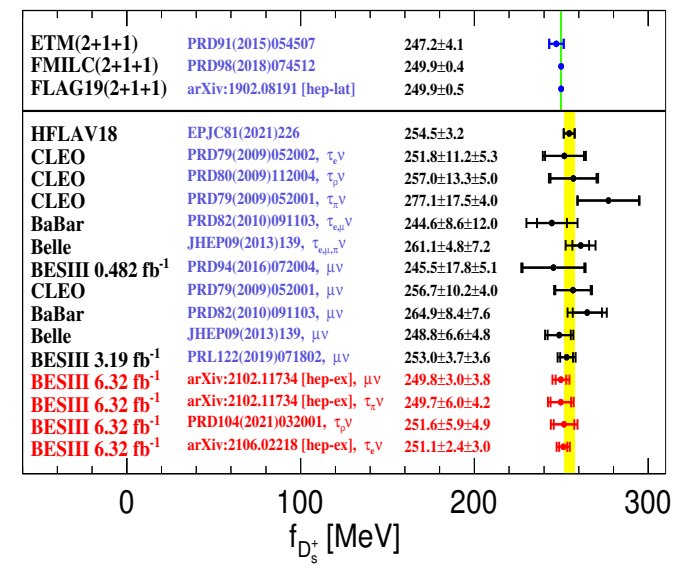

\begin{tabular}{|c|c|c|c|}
\hline CKMFitter & PTEP2020(2020)083C01 & $0.97320 \pm 0.00011$ & $!$ \\
\hline HFLAV18 & EPJC81(2021)226 & $0.969 \pm 0.010$ & - \\
\hline CLEO & PRD79(2009)052002, $\tau_{\mathrm{e}} \mathrm{V}$ & $0.981 \pm 0.044 \pm 0.021$ & $\mapsto-1$ \\
\hline CLEO & PRD80(2009)112004, $\tau_{p} v$ & $1.001 \pm 0.052 \pm 0.019$ & $\mapsto$ \\
\hline CLEO & PRD79(2009)052001, $\tau_{\pi} v$ & $1.079 \pm 0.068 \pm 0.016$ & $\mapsto$ \\
\hline BaBar & PRD82(2010)091103, $\tau_{e, u} v$ & $0.953 \pm 0.033 \pm 0.047$ & $\mathrm{Ho+H}$ \\
\hline Belle & JHEP09(2013)139, $\tau_{\varepsilon, \mu, n^{v}}$ & $1.017 \pm 0.019 \pm 0.028$ & н*1 \\
\hline BESIII $0.482 \mathrm{fb}^{-1}$ & PRD94(2016)072004, $\mu v$ & $0.956 \pm 0.069 \pm 0.020$ & $\mapsto$ \\
\hline CLEO & PRD79(2009)052001, $\mu \nu$ & $1.000 \pm 0.040 \pm 0.016$ & Hי山 \\
\hline BaBar & PRD82(2010)091103, $\mu \nu$ & $1.032 \pm 0.033 \pm 0.029$ & Por \\
\hline Belle & JHEP09(2013)139, $\mu \mathrm{v}$ & $0.969 \pm 0.026 \pm 0.019$ & m \\
\hline BESIII $3.19 \mathrm{fb}^{-1}$ & PRL122(2019)071802, $\mu \nu$ & $0.985 \pm 0.014 \pm 0.014$ & $\omega$ \\
\hline BESIII $6.32 \mathrm{fb}^{-1}$ & arXiv:2102.11734 [hep-ex], $\mu v$ & $0.973 \pm 0.012 \pm 0.015$ & - \\
\hline BESIII $6.32 \mathrm{fb}^{-1}$ & arXiv:2102.11734 [hep-ex], $\tau_{\pi} v$ & $0.972 \pm 0.023 \pm 0.016$ & w \\
\hline BESIII $6.32 \mathrm{fb}^{-1}$ & PRD104(2021)032001, $\tau_{\rho} v$ & $0.980 \pm 0.023 \pm 0.019$ & m \\
\hline BESIII $6.32 \mathrm{fb}^{-1}$ & arXiv:2106.02218 [hep-ex], $\tau_{\mathrm{e}} v$ & $0.978 \pm 0.009 \pm 0.012$ & $m$ \\
\hline & $\left|\mathrm{V}_{\mathrm{cS}}\right|^{0}$ & & 1 \\
\hline
\end{tabular}

Figure 1: Comparisons of decay constant $f_{D_{s}^{+}}$and CKM matrix element $\left|V_{c s}\right|$ between BESIII measurements and other results.

\section{Semi-leptonic $D_{(s)}$ decays}

$3.1 D^{+} \rightarrow \eta \mu^{+} v_{\mu}$

BESIII has reported the first measurement on dynamics of $D^{+} \rightarrow \eta \mu^{+} v_{\mu}$ decay [10]. The measured BF is determined to be $\mathcal{B}_{D^{+} \rightarrow \eta \mu^{+} v_{\mu}}=\left(10.4 \pm 1.0_{\text {stat. }} \pm 0.5_{\text {syst. }}\right) \times 10^{-4}$. Combined with the world average value of $\mathcal{B}_{D^{+} \rightarrow \eta e^{+} v_{e}}$ [8], the ratio of $\mathcal{B}_{D^{+} \rightarrow \eta \mu^{+} v_{\mu}}$ over $\mathcal{B}_{D^{+} \rightarrow \eta e^{+} v_{e}}$ is estimated to be $(0.91 \pm 0.13)$, which is consistent with the SM prediction with a range of (0.97-1.00). Subsequently, the form factor of $f_{+}^{\eta}(0)=0.39 \pm 0.04_{\text {stat. }} \pm 0.01_{\text {syst. }}$, and the CKM matrix element $\left|V_{c d}\right|=0.242 \pm 0.022_{\text {stat. }} \pm 0.006_{\text {syst. }} \pm 0.033_{\text {theory }}$.

3.2 $D^{+} \rightarrow \bar{K}_{1}(1270)^{0} e^{+} v_{e}$

An unbinned extended maximum likelihood fit to the $2 \mathrm{D}$ distributions is performed to analyze the signal $D^{+} \rightarrow \bar{K}_{1}(1270)^{0} e^{+} v_{e}$ with $\bar{K}_{1}(1270)^{0} \rightarrow K^{-} \pi^{+} \pi^{0}$ [11]. Here, the 2D distributions 
are the invariant mass of $K^{-} \pi^{+} \pi^{0}$ and the distribution of the missing energy minus the missing momentum. The obtained signal yield is $(119.7 \pm 13.3)$, and the measured BF is $\mathcal{B}_{D^{+} \rightarrow \bar{K}_{1}(1270)^{0} e^{+} v_{e}}=$ $\left(2.30 \pm 0.26_{-0.21}^{+0.18} \pm 0.25_{\mathrm{ex}}\right) \%$.

3.3 $D^{0} \rightarrow K_{1}(1270)^{-} e^{+} v_{e}$

The signal $D^{0} \rightarrow K_{1}(1270)^{-} e^{+} v_{e}$ with $K_{1}(1270)^{-} \rightarrow K^{-} \pi^{+} \pi^{-}$[12] is analyzed through an unbinned extended maximum likelihood simultaneous fit to the $\mathrm{MM}^{2}$ and the invariant mass of $K^{-} \pi^{+} \pi^{-}$, sharing the same value of the product of $\mathcal{B}_{D^{0} \rightarrow K_{1}(1270)^{-} e^{+} v_{e}}$ and $\mathcal{B}_{K_{1}(1270)^{-} \rightarrow K^{-} \pi^{+} \pi^{-}}$. The signal yield is obtained to be $(109.0 \pm 12.5)$, and the measured BF is $\mathcal{B}_{D^{0} \rightarrow K_{1}(1270)^{-} e^{+} v_{e}}=$ $\left(1.09 \pm 0.13_{-0.13}^{+0.09} \pm 0.12_{\mathrm{ex}}\right) \times 10^{-3}$. Combined with the $D^{+}$case, the ratio of the decay widths of $\Gamma_{D^{0} \rightarrow K_{1}(1270)^{-} e^{+} v_{e}}$ over $\Gamma_{D^{+} \rightarrow \bar{K}_{1}(1270)^{0} e^{+} v_{e}}$ is evaluated to be $\left(1.20 \pm 0.02_{\text {stat. }} \pm 0.14_{\text {syst. }} \pm 0.04_{\mathrm{ex}}\right)$, which agrees with unity as predicted by isospin symmetry.

3.4 $D_{s}^{+} \rightarrow X e^{+} v_{e}$

In order to study the inclusive semielectronic $D_{s}^{+}$decays [13], a fit to the momentum of the positron $\left(p_{e}\right)$ greater than $200 \mathrm{MeV} / c$ is performed, and the signal yield in $p_{e}<200 \mathrm{MeV} / c$ is extrapolated from the fit. The signal region in the whole region of $p_{e}$ is estimated to be $(16648 \pm 326)$, and the measured BF is $\mathcal{B}_{D_{s}^{+} \rightarrow X e^{+} v_{e}}=\left(6.30 \pm 0.13_{\text {stat. }} \pm 0.10_{\text {syst. }}\right) \times 10^{-2}$. The ratio of decays widths of $\Gamma_{D_{s}^{+} \rightarrow X e^{+} v_{e}}$ over $\Gamma_{D^{0} \rightarrow X e^{+} v_{e}}$ is evaluated to be $\left(0.790 \pm 0.016_{\text {stat. }} \pm 0.020_{\text {syst. }}\right)$, which is consistent with the prediction of 0.813 .

\section{Summary}

Utilizing the data samples of $2.93 \mathrm{fb}^{-1}$ at $\sqrt{s}=3.773 \mathrm{GeV}$ and $6.32 \mathrm{fb}^{-1}$ at $\sqrt{s}=4.178$ $4.226 \mathrm{GeV}$, BESIII has reported the purely leptonic and inclusive semielectronic $D_{s}^{+}$decays with higher precision, the first measurement on dynamics of $D^{+} \rightarrow \eta \mu^{+} v_{\mu}$, and the first observations for $D^{+} \rightarrow \bar{K}_{1}(1270)^{0} e^{+} v_{e}$ and $D^{0} \rightarrow K_{1}(1270)^{-} e^{+} v_{e}$ decays. In the near future, BESIII will collect another $17 \mathrm{fb}^{-1}$ at $\sqrt{s}=3.773 \mathrm{GeV}$ and $3 \mathrm{fb}^{-1}$ at $\sqrt{s}=4.178 \mathrm{GeV}$ data samples [14], which will help us to achieve a further improved precision on measurements of the purely leptonic and semi-leptonic $D_{(s)}$ decays.

\section{References}

[1] D. Silverman and H. Yao, Phys. Rev. D 38, 214 (1988).

[2] J. P. Lees et al. (BABAR Collaboration), Phys. Rev. Lett. 109, 101802 (2012); J. P. Lees et al. (BABAR Collaboration), Phys. Rev. D 88, 072012 (2013); R. Aaij et al. (LHCb Collaboration), Phys. Rev. Lett. 113, 151601 (2014); R. Aaij et al. (LHCb Collaboration), Phys. Rev. Lett. 115, 111803 (2015); S. Wehle et al. (Belle Collaboration), Phys. Rev. Lett. 118, 111801 (2017).

[3] M. Ablikim et al. (BESIII Collaboration), arXiv:2102.11734 [hep-ex]. 
[4] R. M. Baltrusaitis et al. (MARK-III Collaboration), Phys, Rev. Lett. 56, 2140 (1986); J. Adler et al. (MARK-III Collaboration), Phys. Rev. Lett. 60, 89 (1988).

[5] M. Ablikim et al. (BESIII Collaboration), Nucl. Instrum. Meth. A 614, 345 (2010).

[6] M. Ablikim et al. (BESIII Collaboration), arXiv:2106.02218 [hep-ex].

[7] M. Ablikim et al. (BESIII Collaboration), Phys. Rev. D 104, 032001 (2021).

[8] P. A. Zyla et al. (Particle Data Group), Prog. Theor. Exp. Phys. 2020, $083 C 01$ (2020).

[9] S. Aoki et al. (Flavour Lattice Averaging Group), Eur. Phys. J. C 80, 113 (2020).

[10] M. Ablikim et al. (BESIII Collaboration), Phys. Rev. Lett. 124, 231801 (2020).

[11] M. Ablikim et al. (BESIII Collaboration), Phys. Rev. Lett. 123, 231801 (2020).

[12] M. Ablikim et al. (BESIII Collaboration), arXiv:2102.10850 [hep-ex].

[13] M. Ablikim et al. (BESIII Collaboration), Phys. Rev. D 104, 012003 (2021).

[14] M. Ablikim et al., Chin. Phys. C 44, 040001 (2020). 